Article

\title{
Piezoresistive Response of Integrated CNT Yarns under Compression and Tension: The Effect of Lateral Constraint
}

\author{
Jude C. Anike, Huy H. Le, Grace E. Brodeur, Mathew M. Kadavan and Jandro L. Abot * \\ Department of Mechanical Engineering, The Catholic University of America, Washington, DC 20064, USA; \\ 09anike@cua.edu (J.C.A.); 55le@cua.edu (H.H.L.); 31brodeur@cua.edu (G.E.B.); \\ mathewmkadavan@gmail.com (M.M.K.) \\ * Correspondence: abot@cua.edu; Tel.: +1-202-319-4382; Fax: +1-202-319-5173 \\ Academic Editor: Craig E. Banks \\ Received: 17 February 2017; Accepted: 29 April 2017; Published: 5 May 2017
}

\begin{abstract}
Carbon nanotube (CNT) yarns are fiber-like materials that exhibit excellent mechanical, electrical and thermal properties. More importantly, they exhibit a piezoresistive response that can be tapped for sensing purposes. The objective of this study is to determine experimentally the piezoresistive response of CNT yarns that are embedded in a polymeric medium while subjected to either tension or compression, and compare it with that of the free or unconstrained CNT yarns. The rationale is the need to know the piezoresistive response of the CNT yarn while in a medium, which provides a lateral constraint to the CNT yarn, thus mimicking the response of integrated CNT yarn sensors. The experimental program includes the fabrication of samples and their electromechanical characterization. The CNT yarns are integrated in polymeric beams and subjected to four-point bending, allowing the determination of their response under tension and compression. The electromechanical data from a combined Inductance-Capacitance-Resistance (LCR) device and a mechanical testing system were used to determine the piezoresistive response of the CNT yarns. At a strain rate of $0.006 \mathrm{~min}^{-1}$, the gauge factor obtained under tension for a maximum strain of $0.1 \%$ is $\sim 29.3$ which is higher than 21.2 obtained under compression. The CNT yarn sensor exhibited strain rate dependence with a gauge factor of approximately 23.0 at $0.006 \mathrm{~min}^{-1}$, in comparison to 19.0 and 1.3 , which were obtained at $0.0005 \mathrm{~min}^{-1}$ and $0.003 \mathrm{~min}^{-1}$, respectively. There is a difference of up to two orders of magnitude in the sensitivity of the constrained CNT yarn under bending with respect to that of the free CNT yarn under uniaxial tension. However, the difference becomes smaller when the constrained CNT yarn was tested under uniaxial tension. This data and information will be used for future modeling efforts and to study the phenomena that occur when CNT yarns are integrated in polymeric and composite materials and structures.
\end{abstract}

Keywords: carbon nanotube yarn; strain sensing; polymer; piezoresistivity; experimental

\section{Introduction}

Since the carbon nanotube (CNT) yarns' discovery in 1991, there have been many researches and experiments about the CNT yarns due to their superb mechanical and electrical properties compared to those of other fibers. Some studies show that the elastic modulus varies from 70 to $350 \mathrm{GPa}$ and the tensile strength from 0.23 to $8.8 \mathrm{GPa}$ [1]. Thus, the elastic modulus of CNT yarns can be greater than that of aluminum (70 GPa) and of steel (210 GPa). Moreover, recent studies also show that the specific conductivity of CNT yarns may reach a maximum of $19.6 \times 10^{6} \mathrm{~S} \mathrm{~m}^{-1} \mathrm{~g}^{-1} \mathrm{~cm}^{3}$ exceeding $14.15 \times 10^{6} \mathrm{~S} \mathrm{~m}^{-1} \mathrm{~g}^{-1} \mathrm{~cm}^{3}$ and $6.52 \times 10^{6} \mathrm{~S} \mathrm{~m}^{-1} \mathrm{~g}^{-1} \mathrm{~cm}^{3}$, which are the specific conductivity of aluminum and copper, respectively [2,3]. The mechanical and electrical properties of CNT yarns could also vary widely depending on their fabrication methods. 
Carbon nanotubes could be single-walled (SWCNT) or multi-walled (MWCNTs) and synthesized by several methods [4]. CNT yarns are composed of many carbon nanotubes produced mostly in four methods: spinning from CNT forest initially grown on a substrate; spinning directly from a CNT aerogel formed from Chemical Vapor Deposition (CVD) process; spinning from a polymer solution containing polyvinylalcohol (PVA); and spinning from CNT films [4-9]. In order to increase compaction within the yarns or the van der Waals forces between individual CNTs, a post-treatment process is required by adding a solvent and evaporating it. Fabrication plays a vital role not only in the quality of the CNT yarns, but also on their properties. Among these four methods, the most popular one is the spinning from a CNT forest. Therefore, the CNT yarns used in this study are formed by this method, which consists of two primary stages: forming a CNT forest on a silicon wafer by CVD reaction and drawing the CNT yarns from it. Long continuous MWCNTs are drawn out and twisted from the CNT forest to form the yarn [4]. The twist naturally produces turns and angles. In general, the higher the angle and the number of turns, the higher the density of the CNT yarns [4]. However, optimization is required because the tensile strength reaches a maximum at a certain value of twisting density and then it decreases [10]. Figure 1 shows an image of the twisted CNT yarn obtained by Scanning Electron Microscope (SEM). After fabrication, the CNT yarn was wound around a spool for storing and handling purposes.

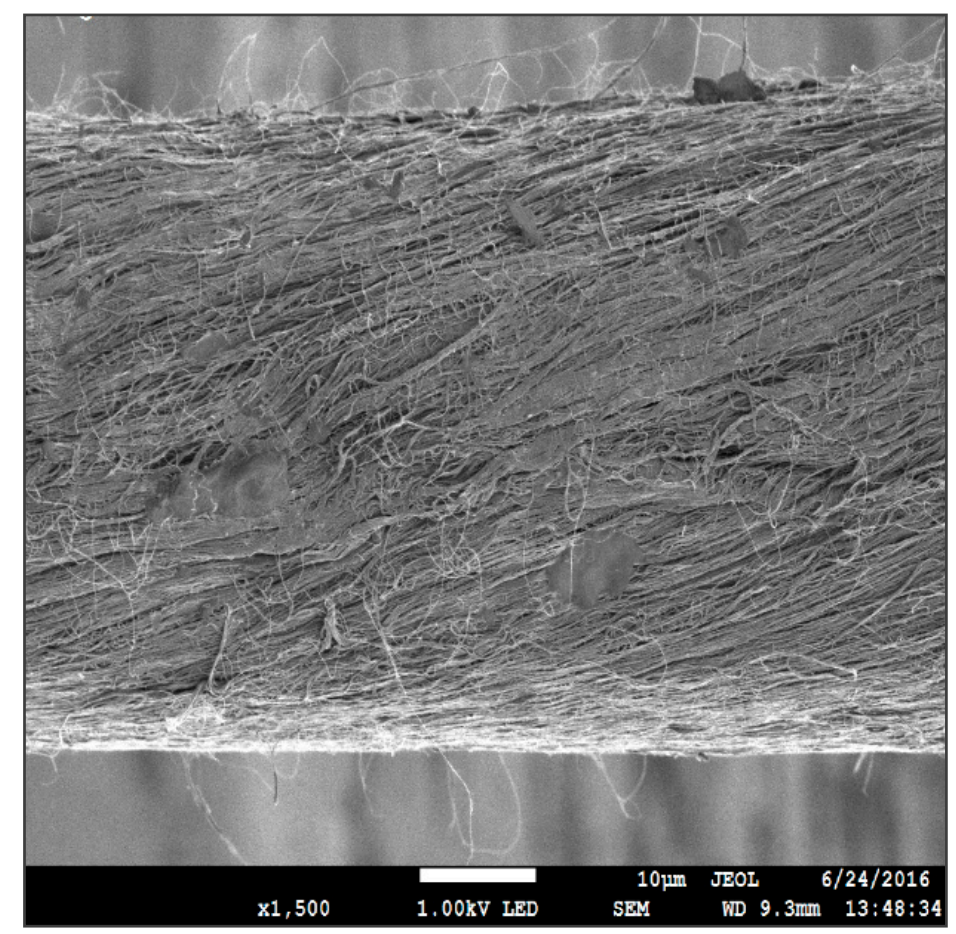

Figure 1. Scanning Electron Microscope (SEM) image of carbon nanotube (CNT) yarn used in experiments (Picture taken using JEOL JSM-7100FA FE SEM).

Piezoresistivity is the most important property of the CNT yarns for their application as sensors [11-18]. From a microstructural perspective, for low strain levels $(<2 \%)$, metallic nanotubes play a vital role in the change of the electrical resistance $[1,11]$. For a high strain level, at $2.5 \%$, semiconducting nanotubes dominate the change in the resistance of the CNT yarns [12,13]. The recent research shows that there are two underlying phenomena that take place at different strain rates [12]. It is said that the bundles of carbon nanotubes untwist during loading and contact length decreases (the density of CNT yarns per unit length decreases), which leads to the increase in resistance. In contrast, the electrical resistance decreases during the unloading segments $[12,14,15]$. In addition, there is a decrease in resistance that occurs due to inter-tube/inter-bundle slippage (inelastic shear motion) 
caused by yarn's relaxation and structural reformation during the loading segments, and a continuous decrease in resistance occurs during unloading as the yarn recovers its (conductive) structure [16]. Based on previous studies, the first phenomenon, whereby a resistance increase is recorded during the loading and a decrease in resistance in the unloading segments is dominant at high strain rates. At a very low quasi-static strain rates, a resistance decrease during both loading and unloading segments has been reported [16]. Presented next is the study of the piezoresistive response of the CNT yarns integrated in a polymeric medium and its comparison with that of the free or unconstrained CNT yarns.

\section{Materials and Fabrication}

\subsection{Carbon Nanotube Yarn}

The CNT yarn used in this study is a one-thread yarn grown from a vertically aligned CNT array with no post-processing at The Nanoworld Laboratories, University of Cincinnati. A $10 \mathrm{~cm}$ Si wafer having a $5 \mathrm{~nm}$ alumina $\left(\mathrm{Al}_{2} \mathrm{O}_{3}\right)$ buffer layer was used with an iron-based $1.2 \mathrm{~nm}$ thin film catalyst, both magnetron sputtered. The dicing process on the Si wafer substrates were performed by scribing and breaking into $5 \mathrm{~cm}$-long and variable width (up to $3.75 \mathrm{~cm}$ ) substrates, loaded into a CVD reactor (ET3000) from CVD Equipment Corporation. In the presence of Ar, the reactor was heated up to $400{ }^{\circ} \mathrm{C}$ for annealing. After $2 \mathrm{~min}$, the temperature of the reactor was ramped to $750{ }^{\circ} \mathrm{C}$, then a $300 \mathrm{sccm}$ mixture of $\mathrm{C}_{2} \mathrm{H}_{4}$ and $1000 \mathrm{sccm}$ of Ar was introduced for about $20 \mathrm{~min}$. The growth was achieved at a pressure of $98.7 \mathrm{kPa}$. Upon growth completion, $30 \mathrm{sccm}$ of $\mathrm{H}_{2} \mathrm{O}$ and $2000 \mathrm{sccm}$ of Ar were delivered during cooling to promote CNT array detachment [17].

The as-spun CNT array was approximately $350-400 \mu \mathrm{m}$ in height, with a distribution of one up to six or seven walls. The array was spun into yarn at about $2000 \mathrm{rpm}$ twisting rate and about $320 \mathrm{~mm} / \mathrm{min}$-pulling speed, giving a yarn with an angle of twist of about $30^{\circ}$. Densification was achieved with acetone. The CNT yarn's diameter is about $25-30 \mu \mathrm{m}$. The measured average resistivity of the CNT yarn used in this study is about $1.7 \times 10^{-3} \Omega \mathrm{cm}$.

\subsection{Fabrication}

The CNT yarns were integrated into polymeric samples cast using a silicon rubber mold. Lines were drawn on the back of the mold for precision in integration of both wires and CNT yarn. Then the electrical copper wires were integrated into the mold. The wires were threaded through needles, and then stitched through the mold at the pre-marked positions. Due to their stiffness, the wires were stitched to go under the CNT yarns to eliminate any stress which may be caused by the contact between the wires and the CNT yarn during fabrication. Conductive paint, Bare Paint ${ }^{\mathrm{TM}}$, was used to connect the CNT yarns to the copper wires.

The following step consisted of integrating the CNT yarns into the mold. The CNT yarn was removed gently from the spool. Secondly, a sharp scalpel or scissor was used to make a clean cut to separate the yarn from its original batch. To ensure that the prepared CNT yarn was inside the mold completely, the length of CNT yarn needed to exceed the length of the rubber mold. The CNT yarns were threaded through a needle, then taped down at the two ends that are outside the mold to ensure that it is straight. Two tweezers were used during this process. Their precision and small tips allow for more accuracy in handling to avoid any damage to the CNT yarn. The CNT yarns were stitched through the mold as shown in Figure 2a. To preserve the straightness of the CNT yarn, it is lightly pre-tension and then held down using a Scotch ${ }^{\mathrm{TM}}$ tape. Finally, the conductive paint was applied using a syringe to provide a better control of the amount of paint on the connection points.

The dimensions of the sample are important, especially in a four-point bending setup to accommodate the strain gauges at the central section where pure bending is required (Figure $2 b$ ). The matrix materials for the samples are: EPON ${ }^{\mathrm{TM}}$ Resin 862 (Diglycidyl Ether of Bisphenol F) epoxy resin and Epikure W curing agent, both from Miller-Stephenson Chemical Co., (Danbury, CT, USA). With a mix ratio of 1:0.264 of resin and hardener, the two chemicals were weighed and mixed in a 
beaker for $3 \mathrm{~min}$. Next, the mixture was heated to $130{ }^{\circ} \mathrm{C}$ for $15 \mathrm{~min}$ to eliminate air bubbles. The fully mixed solution was poured into the mold with integrated electrical wires and CNT yarns and the mold inserted into a Tetrahedron press machine for curing using a 4-h 2-step heating cycle. During the first $2 \mathrm{~h}$, the temperature was set to $100^{\circ} \mathrm{C}$ and $130{ }^{\circ} \mathrm{C}$ for the last $2 \mathrm{~h}$. A pressure of about $0.55 \mathrm{MPa}$ was applied to the mold during the curing cycle. Figure $2 \mathrm{c}$ shows the final samples after curing them during $4 \mathrm{~h}$ and cut into halves. The thickness of the beam is $9 \mathrm{~mm}$ and the CNT yarn is about $0.5 \mathrm{~mm}$ from the surface. Strain gauges are mounted on each side of the sample as shown in Figure $2 \mathrm{~d}$.
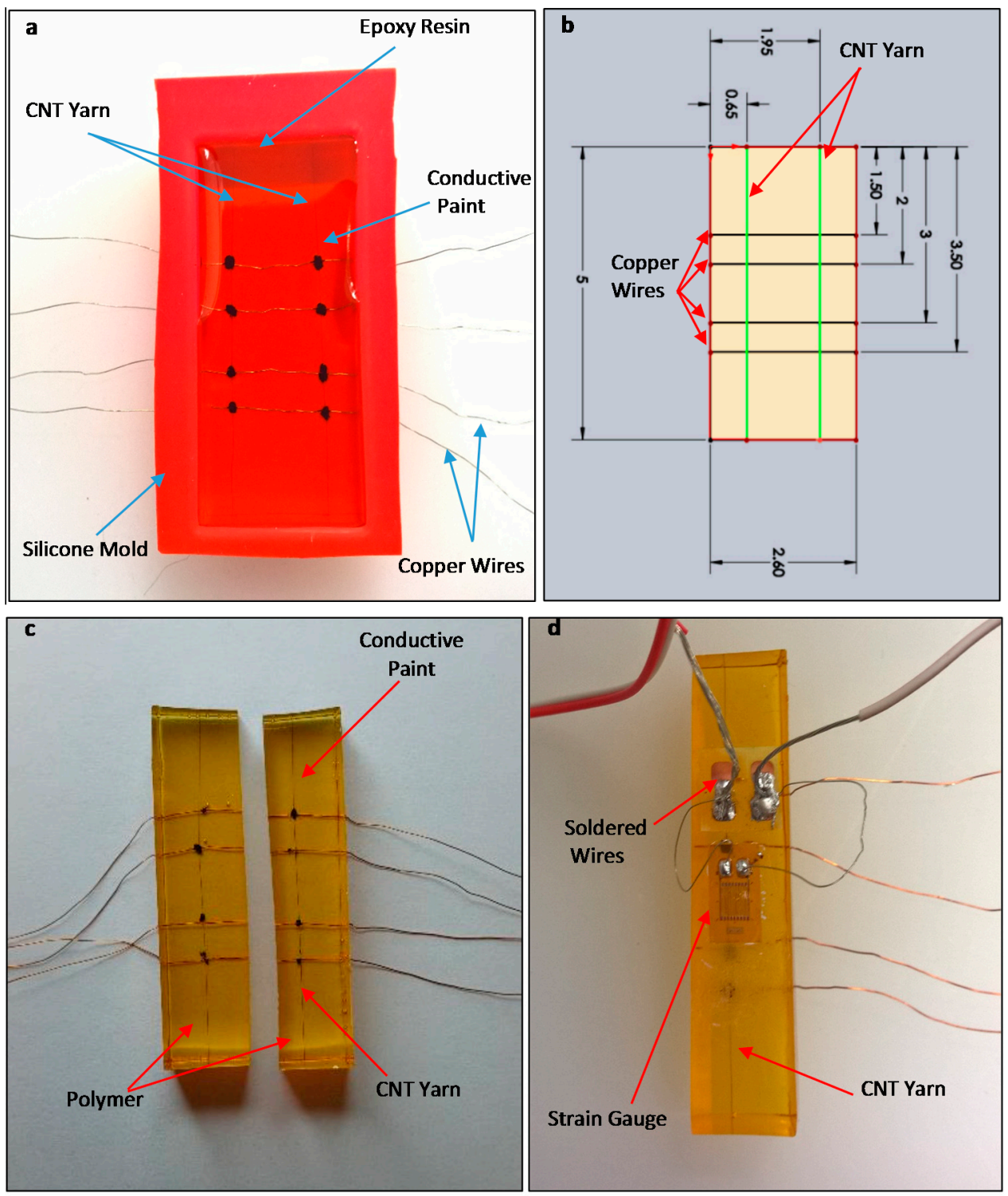

Figure 2. Optical images: (a) Silicone rubber mold and CNT yarns bonded to the wires with conductive paint prior to pouring the polymer; (b) Schematic of the sample with dimensions in $\mathrm{cm}$; (c) Fabricated samples with instrumented wires; (d) Sample with strain gauge mounted on it.

\subsection{Mechanical and Electrical Measurements}

To determine the mechanical response of the CNT yarns, an MTS ${ }^{\mathrm{TM}}$ Criterion 43 electromechanical loading machine was used along with a 30-kN-capacity load cell to provide the load and record all the mechanical data from the samples. Simultaneously, the electrical response was recorded by an electrical data acquisition system. As mentioned previously, there are four copper wires integrated into polymer beam for electrical connection. These electrical wires were connected to a National Instruments 
PXI-4072 Inductance-Capacitance-Resistance (LCR) reader unit mounted in a National Instruments PXI-1033 chassis via two Bayonet Neill-Concelman (BNC) cables with Mini-hooks electrical leads. The LCR's maximum resistance measurement is $10 \mathrm{M} \Omega$. The data acquisition rates of both the LCR and the MTS system were $1 \mathrm{~Hz}$. Strain rates of $0.0005,0.003$ and $0.006 \mathrm{~min}^{-1}$ were used for both tension and compression tests in this study. The electrical measurements from the LCR reader were outputted through a LabVIEW ${ }^{\mathrm{TM}}$ virtual instrument. Figure 3 shows the experimental setup for the experiments.
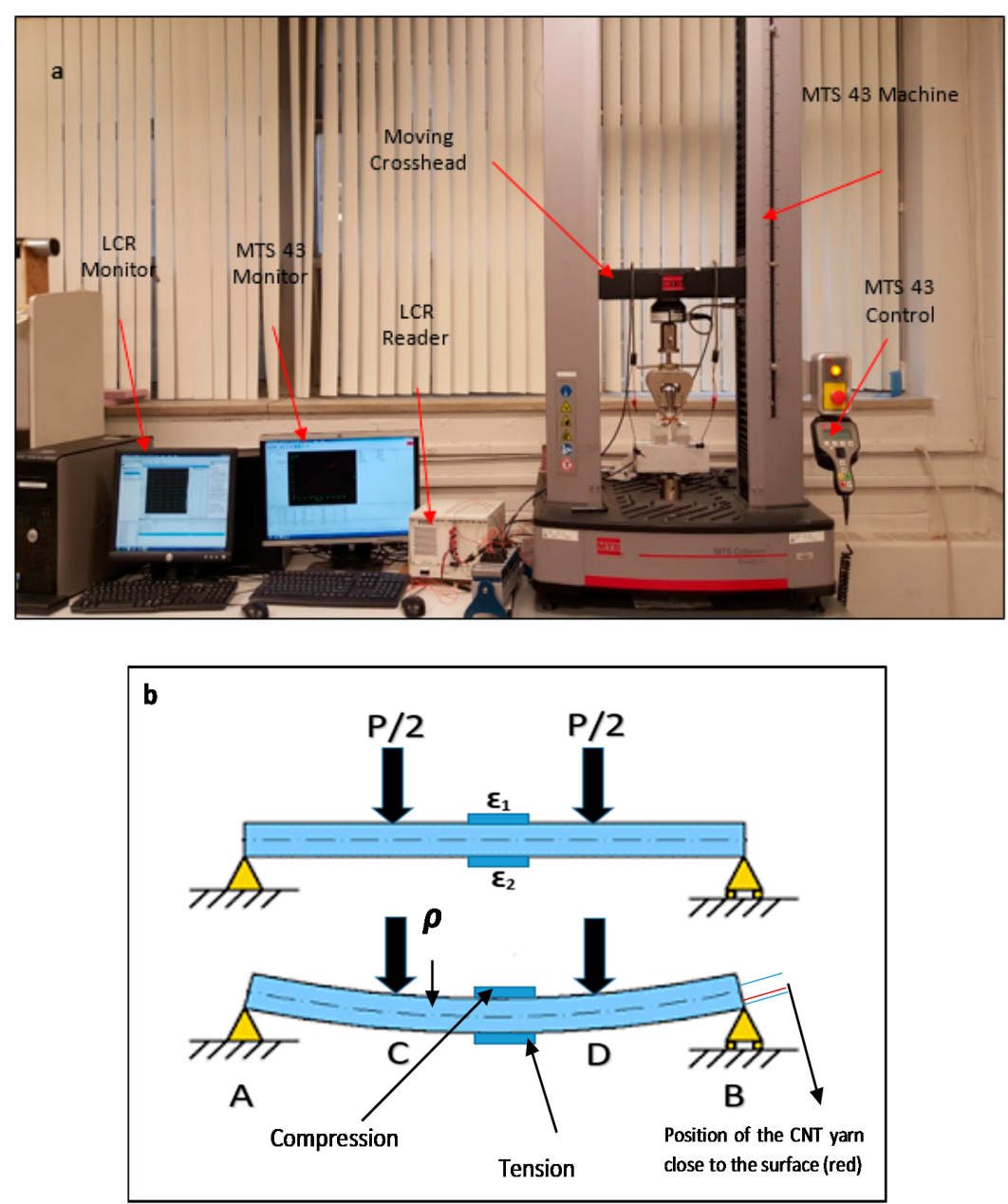

Figure 3. (a) Optical image of experimental setup; (b) Schematics of four-point bending setup of the sample with strain gauges bonded to each side (dimensions: length $=5 \mathrm{~cm}$, width $=1.2 \mathrm{~cm}$, thickness $=0.9 \mathrm{~cm})$.

\subsection{Methodology}

The CNT yarns were embedded in polymeric beams and subjected to four-point bending. The samples were inserted into the loading machine as shown in Figure 3a. To insert samples into the loading machine, a set of fixtures was specially made for these experiments. The upper fixture consists of a square aluminum bar with two small round aluminum dowels. The lower fixture consists of an aluminum block with a track on it. The testing frame was slowly moved downward while the lower fixture was unmoved. It is of utmost importance to keep the electrical wires separated from each other so that they do not touch any metallic parts of the loading machine system or the four-point bending fixture. Since these wires and metals can be conductive, the resistance would be affected by their physical contacts.

The experimental setup allows pure bending in the central section of the simply supported rectangular polymeric beam with supports at points A and B (Figure $3 \mathrm{~b}$ ). A $30 \mathrm{kN}-\mathrm{load}$ cell of the 
loading machine is used to apply a load, $\frac{P}{2}$, at points $C$ and $D$, which are spaced equidistantly from the end and the center of the beam. Two strain gauges are bonded at the center of the top and bottom surfaces of the beam. The strain gauge at the top measures compressive strain, $\varepsilon_{1}$, from bending and conversely; the strain gauge at the bottom measures the tensile strain, $\varepsilon_{2}$. Thus, pure bending occurs between the loading application points, $\mathrm{C}$ to $\mathrm{D}$, where there is a constant bending moment of $M=\frac{P d}{2}$ with a radius of curvature $\rho$. A relation between $M$ and $\rho$ can be used to compute the stress and strain and compare the latter to the values measured from the strain gauges. Since the CNT yarn is embedded on one side alone, close to the surface, it can be assumed that the strain measured by the strain gauge is the one experienced by the CNT yarn. Polymers are homogeneous materials, and thus the strains on the top and bottom surfaces should be identical. However, the side of the polymeric beam with the CNT yarn may experience higher strains due to cracks and cavities created by its integration.

To measure the effect of lateral constraint, additional tests using ASTM D638 specification for the determination of the tensile properties of unreinforced and reinforced plastics in the form of standard dumbbell-shaped test samples were employed. The CNT yarn was embedded in the middle at about half the thickness of a $3.5 \mathrm{~mm}$-thick sample. The same mix ratio used for the polymeric beam was applied to the sample. The sample was cured for $2 \mathrm{~h}$ and post-cured for an additional hour. The dimensions of the sample are presented in Figure 4.
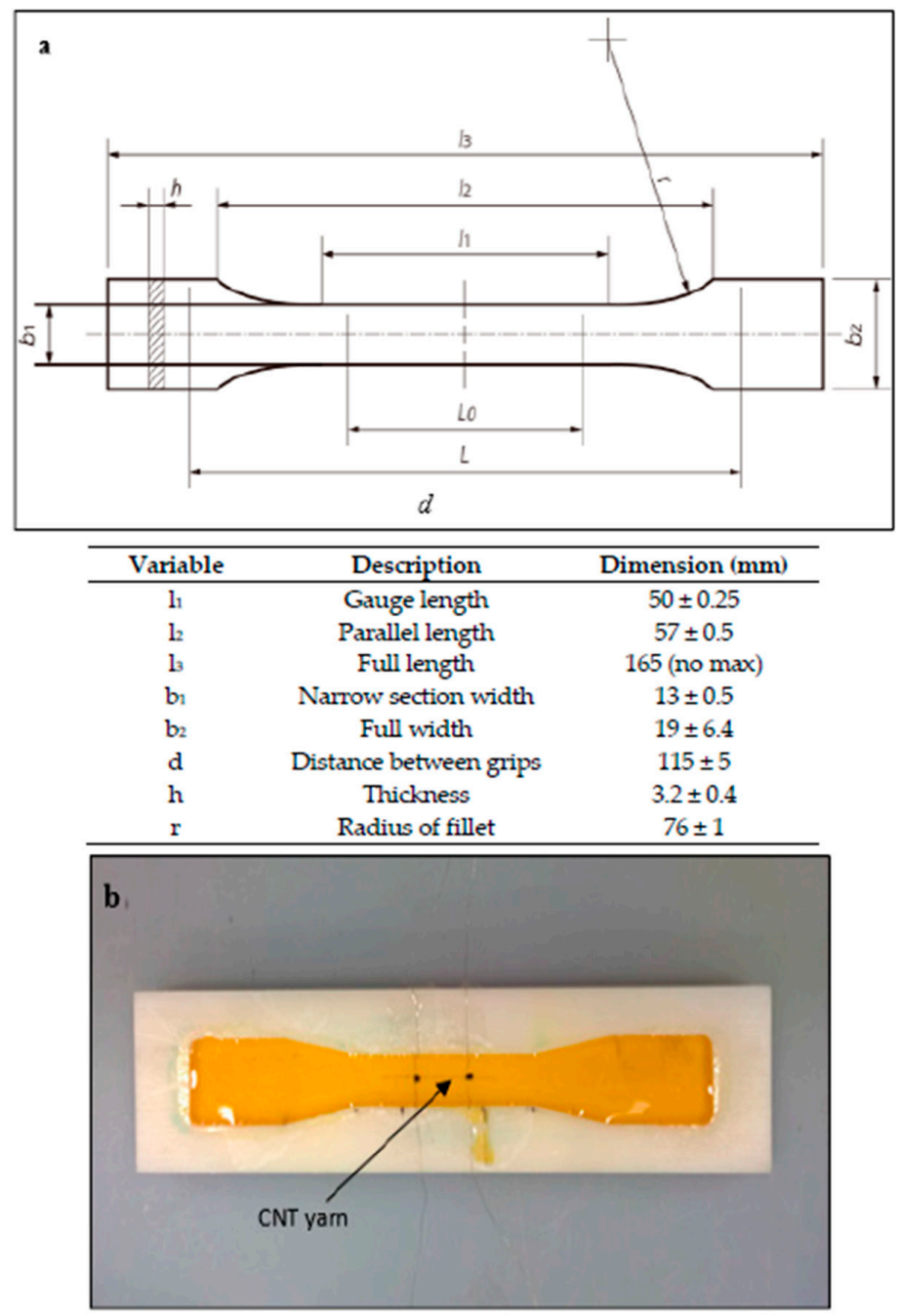

Figure 4. (a) Schematic diagram and dimensions of sample (ASTM D638); (b) Optical image of sample [18]. 


\section{Piezoresistive Response of Integrated CNT Yarns}

The piezoresistive response of the CNT yarns integrated in polymeric beams is presented next. It is worth mentioning that the strain is obtained directly from the bonded strain gauges, which may be a little bit different from that determined from the applied load using a beam theory formulation. The mechanical properties of the CNT yarn can vary across the radial length, so the tests were performed from the CNT yarn cut from the same section and batch. The results in Sections 3.1 and 3.2 correspond only to the bending tests where the CNT yarns are under tension, and similarly in Section 3.3, under compression. In Section 3.4, the results under tension are measured in both bending and uniaxial tensile tests.

\subsection{Piezoresistive Response under Tension}

The embedded CNT yarn in the polymer is constrained by the polymer medium upon solidification. This constraint can affect the diameter of the CNT yarn across its axial length as most polymers shrink during curing. Even for polymers with positive coefficient of thermal expansion (CTE), the rigid structure of the cured polymer allows the CNT yarn to experience better tensile effect as CNT bundles do not slide under tensile force. Theoretically, it is assumed that any decrease in the diameter or increase in length will lead to an increase in the resistance of the CNT yarn per the relation:

$$
R=\rho \frac{L}{A}
$$

where $R$ is the resistance of the CNT yarn, $\rho$ is the electrical resistivity; $L$ and $A$ are the length and cross-sectional area, respectively. When the CNT yarn is embedded in a polymer medium, it acts to constrict the structure of the integrated material. Thus, naturally, both lateral and tensile deformations are limited, making the Poisson's effect less feasible. However, the CNT yarns, just like most fibers, are highly porous [19-24]. Polymers with relatively high molecular weight may infiltrate the fiber but not the inner structure or affect the fiber alignment [25]. Low molecular weight polymer chains will infiltrate the fiber, expand the bundle and intercalate between nanotubes, thus blocking the load and charge-carrying channels, reducing significantly the piezoresistivity. This also depends on the viscosity and surface tension of the polymer. The cross-linked EPON 862 epoxy resin has high molecular weight and viscosity but good wettability. The choice of the matrix was based on its structural stability and to prevent fiber infiltration. Table 1 shows the resistance of the CNT yarn prior and after integration in the nanocomposite beams made with two different epoxy resins and a CNT yarn beaded in a cyanoacrylate adhesive. EPON 862/Epikure W epoxy resin cured per the manufacturer's recommendation showed a better preservation of the resistance value of the CNT yarn compared to other adhesives used. The decrease in resistance is attributed to be related to the CTE of the fiber composite. CNT yarn dip-coated in the same epoxy resin (Epon 862/Epicure W resin) and cured at the same temperature showed an increase in resistance like in the other adhesives. It is suggested that nanotubes, due to their sp2 carbon network within the graphene sheet, have a negative CTE. With a reported axial CTE of $-1.6 \times 10^{-6} \mathrm{~K}^{-1}$ [26], CNT fibers/yarns appear to be a good reinforcement to decrease the thermal expansion of epoxy resin. It is expected that increasing the fiber volume will substantially decrease the CTE of the fiber composite. From the manufacturer's data sheet, the glass transition temperature, $\mathrm{T}_{\mathrm{g}}$ of Epon 862 resin is at $105^{\circ} \mathrm{C}$. Below $\mathrm{T}_{\mathrm{g}}$, the resin molecules lack mobility, resulting in longer time to reach equilibrium [27]. However, the CTE of polymers generally increases when heated and above $\mathrm{T}_{\mathrm{g}}$, an expansion of the polymer molecules is expected. Thermodynamically, a resultant shrinkage arises when the molecules equilibrate and adjust back to initial state. 
Table 1. Resistance measurements of CNT yarn dip-coated in various adhesives.

\begin{tabular}{ccc}
\hline Polymer & Resistance of Pristine CNT Yarn (k $\Omega$ ) & Resistance of CNT Yarn after Curing (k $\Omega$ ) \\
\hline Epon 862/Epicure W Resin (sample 1) & 1.025 & 0.752 \\
Epon 862/Epicure W Resin (sample 2) & 1.200 & 0.884 \\
Epon 862/Epicure W Resin (sample 3) & 1.097 & 0.812 \\
Epon 862/Epicure W Resin (Dip-coated) & 1.183 & 1.328 \\
Loctite & & 1.197 \\
(Epichlorohydrin-4,4'-isopropylidene Diphenol) resin & 0.924 & 1.083 \\
Loctite $^{\mathrm{TM}}$ 454 (Ethyl 2-cyanoacrylate) adhesive & 0.722 & \\
\hline
\end{tabular}

The constriction of the CNT yarn prevents the unbundling or fiber unraveling, which is normally observed in a free or unconstrained CNT yarn. Also, loosening of twists and fiber sliding associated with changes in geometrical properties of the CNT yarn is limited. Thus, the polymer keeps the yarn in place and compact during tensile deformation, providing more surface area for load bearing. It is then expected that the CNT yarn constrained in this medium will be more sensitive to changes in resistance than a free CNT yarn due to the increased surface contact for load transmission. The maximum stress, $\sigma$, in the sample was calculated using beam theory:

$$
\sigma=\frac{M c}{I}
$$

where $I=\frac{b t^{3}}{12}$ for a rectangular beam sample, $M$ and $c$ are the bending moment in the beam and the distance from the neutral axis to the surface respectively, $b$ and $t$ are the width and thickness of the beam, respectively. The corresponding maximum strain value is then calculated from the stress using Hooke's law $\left(\varepsilon=\frac{\sigma}{E}\right)$, to be compared with the strain gauge values.

The cured sample with the integrated CNT yarn has an elastic modulus of about $3.3 \mathrm{GPa}$ in the linear region (Figure 5a). Figure $5 b$ shows the piezoresistive response of a sample under tension. At $0.1 \%$-strain, a gauge factor of approximately 29.3 was obtained. This is a conformation of the expected response but also incredibly high for a low strain level and much higher than the gauge factors of metallic foil strain gauges. The gauge factors obtained for the unconstrained CNT yarn of the same batch of CNT yarn were lower than 1 . This also validates the postulated effect of slippage and fiber disintegration of the free CNT yarns under tension. The relative resistance-strain curve of three other samples tested exhibit a gauge factor in the range of 23 to 30 with an average value of about 26 (Figure 5c).
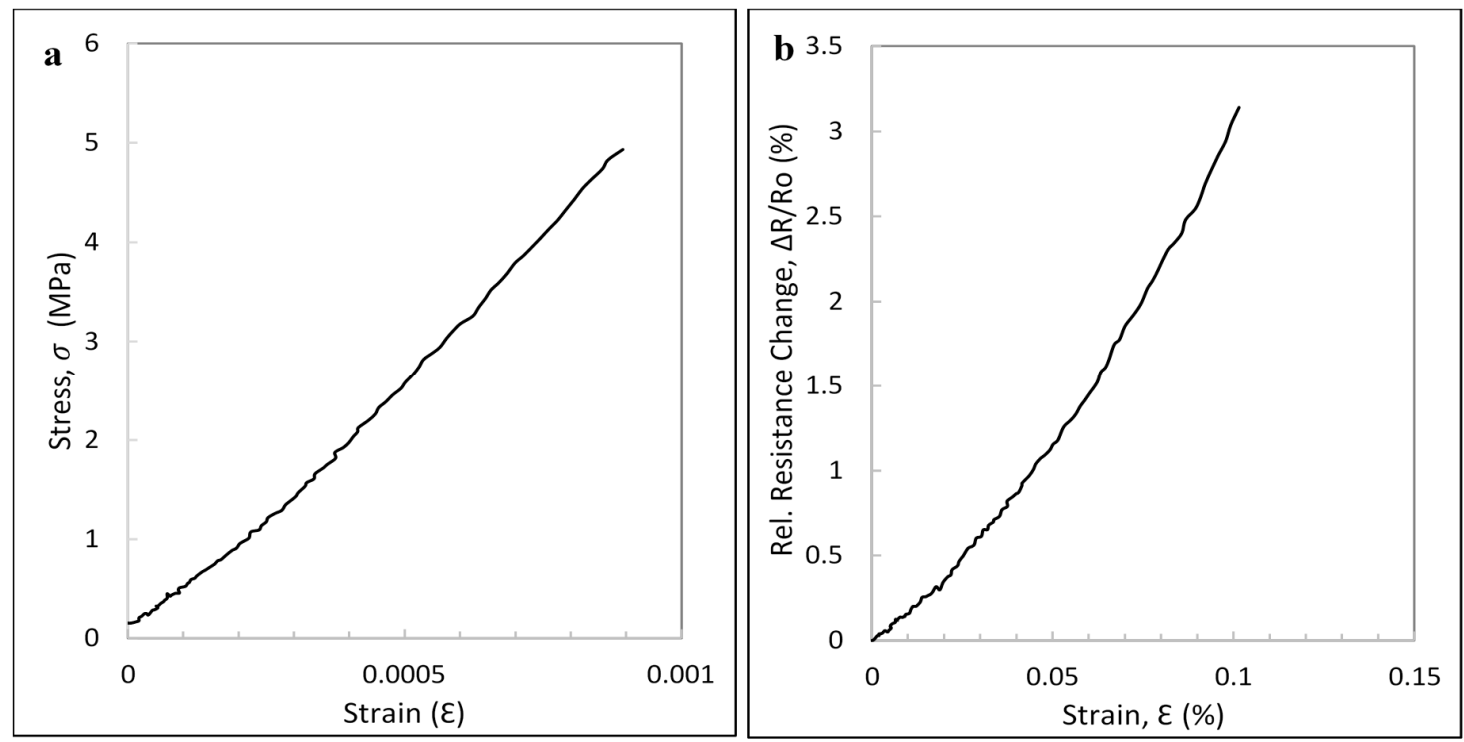

Figure 5. Cont. 


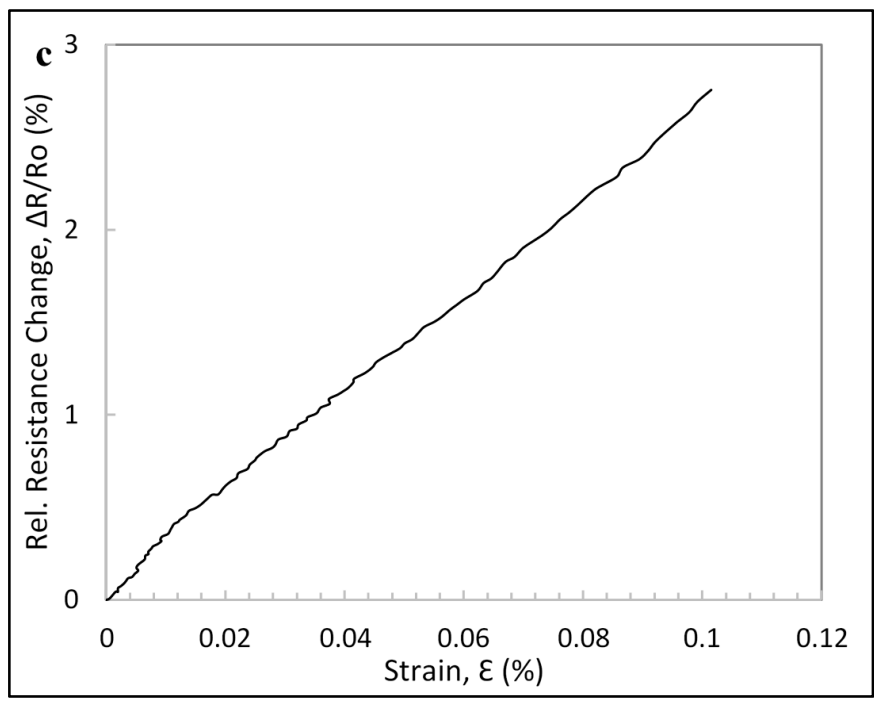

Figure 5. (a) Stress-strain curve of the polymer sample with integrated CNT yarn; (b) Relative resistance change-strain curve of the CNT yarn under tension at a maximum strain of $0.1 \%$ and a strain rate of $0.006 \mathrm{~min}^{-1}$; (c) Average relative resistance change-strain curves of three CNT yarns under tension at a maximum strain of $0.1 \%$-strain and a strain rate of $0.006 \mathrm{~min}^{-1}$.

\subsection{Rate Dependent Effects}

To measure the impact of the strain rate as reported previously [12], the results obtained at different strain rates, $0.0005,0.003$ and $0.006 \mathrm{~min}^{-1}$, were graphed in Figure 6. At a very low strain rate of $0.0005 \mathrm{~min}^{-1}$ and below, the rate sensitivity effect is not so conspicuous in a constrained CNT yarn as it was the case in free CNT yarns. Due to the very significant disparity between the results from the constrained CNT yarn at $0.006 \mathrm{~min}^{-1}$ to both 0.0005 and $0.003 \mathrm{~min}^{-1}$, the strain level was kept at $0.05 \%$. A noticeable nonlinearity was observed as the strain rate was reduced. Figure $6 \mathrm{~b}$ has been smoothened only for the strain rate of $0.0005 \mathrm{~min}^{-1}$ to obtain more insight from the curve. The nonlinearity also affects the values of the gauge factors as any linear trend imposed on the graph will most likely consider peak and valley points, which result from the amplification of background noise at low strain rates. In comparison to Figure $6 \mathrm{~b}$, there is no observable negative piezoresistivity at the low quasi-static strain rates. It is observed that the gauge factors increased significantly when the strain rate reached $0.006 \mathrm{~min}^{-1}$. At $0.006 \mathrm{~min}^{-1}$, the obtained gauge factor was approximately 23.0 in comparison to 19.0 and 1.3 , which were obtained at 0.0005 and $0.003 \mathrm{~min}^{-1}$, respectively. With an increase in strain rate, the linearity of the piezoresistive response improved. It is important to emphasize that a linear response is imperative for practical sensing applications of these CNT yarns.

These results could be explained by the limitation of slippage (inter-fiber/inter-bundle) encountered in a constrained medium. In a free state, the CNT yarn under low strain rate loading experiences slippage, with the effect causing the CNTs to rearrange their structure with the loading. Depending on the rate of loading, the fiber rearrangement can either equalize the rate of deformation leading to a weak load transfer mechanism and mild slippage, or outweigh the loading rate leading to a high slippage effect. It is worth recalling that slippage accounts for negative piezoresistivity in the free CNT yarns [16]. 


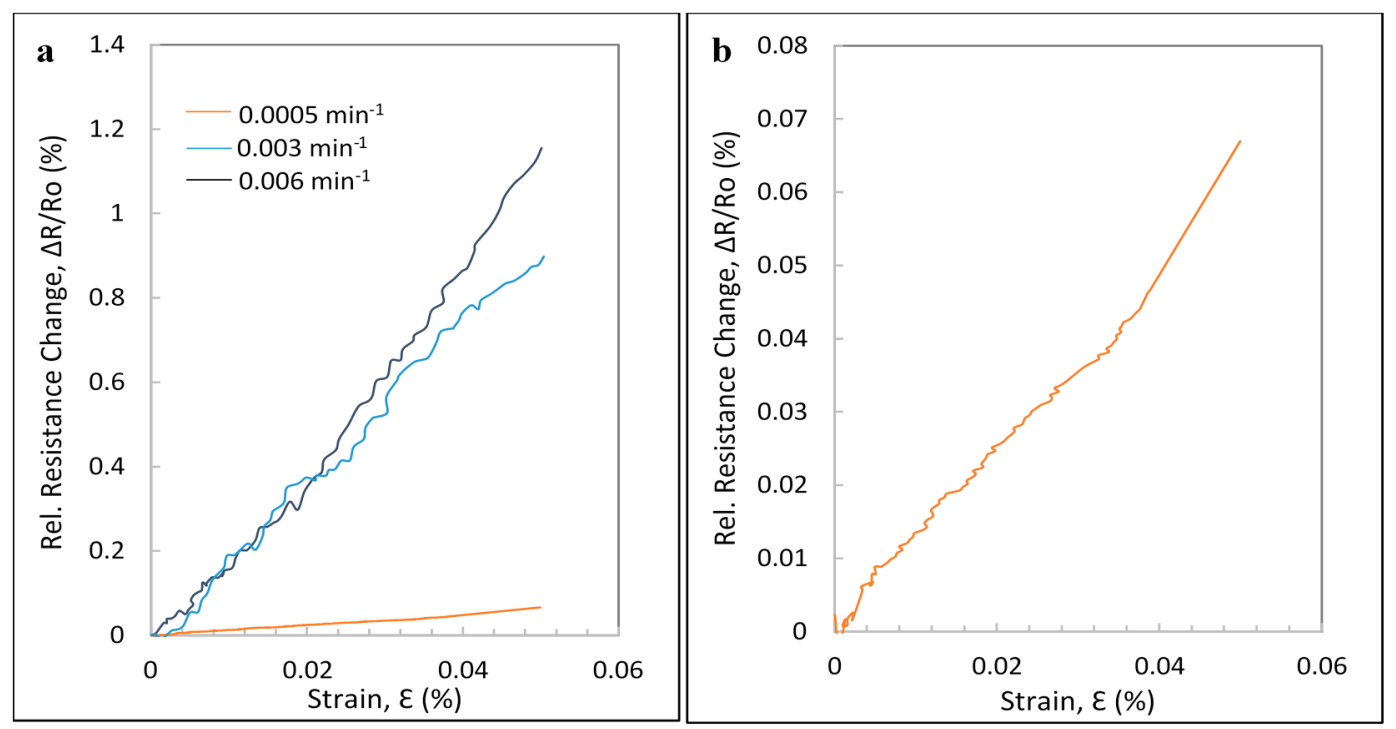

Figure 6. Relative resistance change-strain curves of samples under tension at various strain rates: (a) $0.0005,0.003$ and $0.006 \mathrm{~min}^{-1}$; (b) $0.0005 \mathrm{~min}^{-1}$.

The strain rate sensitivity experienced by the CNT yarn in the constrained medium (polymer), can only be explained by the effect of slippage. Although the polymer medium serves as a constraint to the fiber bundle sliding movement in the CNT yarn, it does not entirely stop slippage. As the strain rate progressively decreased, the effect of slippage became increasingly pronounced. The difference in the piezoresistive response between the constrained and free CNT yarn is that the yarn does not experience enough slippage at low strain rate in a constrained state to affect relaxation due to new structure formation. This is because the polymer medium does not give the CNT yarn bundles enough room for such reformation process, unlike in a free condition.

\subsection{Piezoresistive Response under Compression}

Figure 7a shows the piezoresistive response of the constrained CNT yarn under compression for a maximum strain of $0.1 \%$ and at a strain rate of $0.006 \mathrm{~min}^{-1}$. The value obtained for the gauge factor $(\sim 21.2)$ is significantly lower than in tension ( 29.3) unlike the observed piezoresistive response in carbon fibers [28]. Even increasing the strain up to $0.3 \%$ (Figure $7 b$ ), the obtained gauge factor value of 28.8 is a bit smaller than that obtained at a strain of $0.1 \%$ in tension (Figure 5). The increase in contact area due to an increased fiber volume fraction under compression will result in a significant decrease in resistance change with respect to that of the same strain level in tension. Also, since contact resistance accounts for most of the piezoresistivity of the CNT yarn, the frictional motion is restricted under compression. The high gauge factor signifies that the CNT yarn, with its weak carbon-to-carbon bonds, due to CNTs not spanning the sample length, experiences change in resistance mostly from contact resistance or contact area. However, it is assumed that inclusion of the CNT yarn in the polymer affects its homogeneity as the yarn may become a source for microvoids that may coalesce during loading. Thus, the applied load to achieve the same strain may vary across samples under tension and compression. There is also a good repeatability as seen in Figure 7c. For four samples tested under compression at the lower strain rate of $0.003 \mathrm{~min}^{-1}$, the gauge factor values were 2.2, 2.3, 2.7, and 2.1, respectively, with an average gauge factor of 2.3 and a standard deviation of 0.046 .

Due to the delicate nature of the CNT yarn, it is not possible to test the unconstrained CNT yarn under uniaxial compression to compare its results with those of the constrained CNT yarn. However, it can be deduced from the tensile tests that the constraint imposed by the medium on the CNT yarn would serve to improve stress redistribution and piezoresistive properties. 

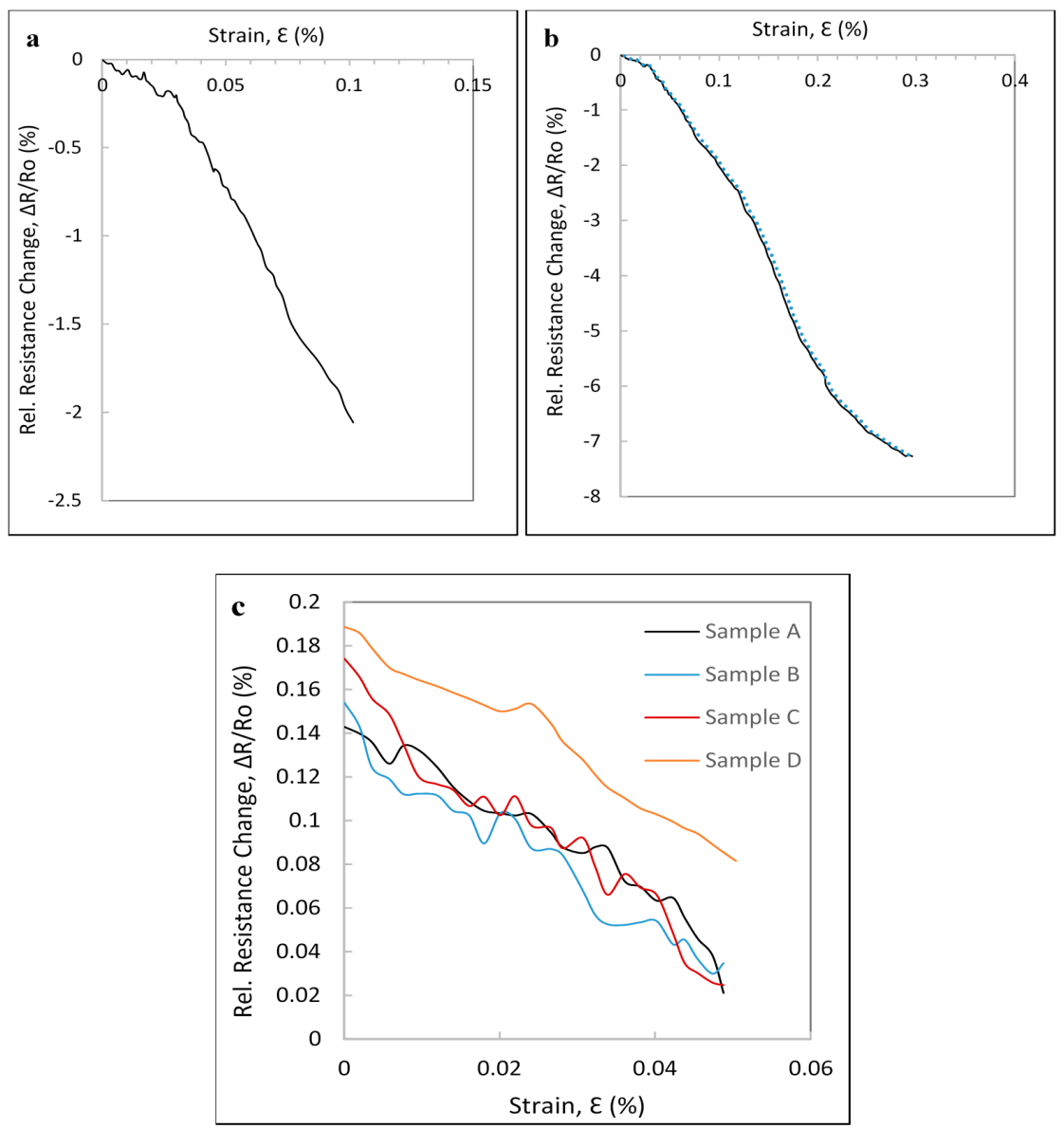

Figure 7. Relative resistance change-strain of constrained CNT yarn samples under compression: (a) 0.1\%-maximum strain; (b) 0.3\%-maximum-strain; (c) 0.05\%-maximum strain (four samples-A, B, C, D).

\subsection{Effect of Lateral Constraint}

Figure 8 shows a comparison of the relative resistance change-strain curves of both the free CNT yarn [12] and the constrained CNT yarn of this study. There is a significant difference in the piezoresistive responses of the constrained and unconstrained CNT yarns [29]. For the samples tested to a maximum strain of $0.1 \%$ at a strain rate of $0.003 \mathrm{~min}^{-1}$, the gauge factors of the constrained yarns under pure bending and pure tension are approximately 15.2 and 2.7, respectively (Figure 8a) compared to 0.1 obtained for the free CNT yarn. The assumption is that the flexural stiffness and strength would be the same as the tensile stiffness and strength, respectively on homogeneous materials. However, in terms of any defect that could be associated with embedding the CNT yarn in the polymer and its position in the sample, there is an expected variability due to testing methods leading to a significant difference in the gauge factors obtained in both testing methods. From Figure 8b, a gauge factor of 30.7 was recorded for the constrained CNT yarn under bending versus 0.2 for the free CNT yarn when tested up to a maximum strain of $0.1 \%$ at a strain rate of $0.006 \mathrm{~min}^{-1}$. The values of the average gauge factors obtained at the $0.05 \%$ and $0.1 \%$ strains are presented in Table 2 . The gauge factor increased from the free to the constrained CNT yarns but it increased much more significantly in the case of the constrained CNT yarn under bending at higher strain. For both conditions, when the strain rate was increased doubly, the value of the gauge factor was twice that at the lower strain rate. However, the strain rate does not affect the value of the gauge factor as much in a free CNT yarn when compared to a constrained CNT yarn [16]. The curves of the constrained CNT yarns are also very linear and produce higher gauge factors than that of the free CNT yarn. Consequently, the piezoresistivity of the constrained CNT yarns is higher than that of the 
unconstrained CNT yarns. Due to the discontinuous nature of the CNTs in a yarn, most of the yarn's resistance is produced by the contact resistance of the individual CNT and their bundles. It is assumed that the fiber volume fraction increases due to the constraint imposed on the CNT yarn by the polymer medium and could lead to a lateral contraction. Thus, there is an increased CNT density in the medium compared to the case of the free CNT yarn. Furthermore, the constraint negates the impact of slippage, thus enhancing inter-fiber load transfer. A more uniform load distribution in the CNT yarn would account for the higher piezoresistive response.
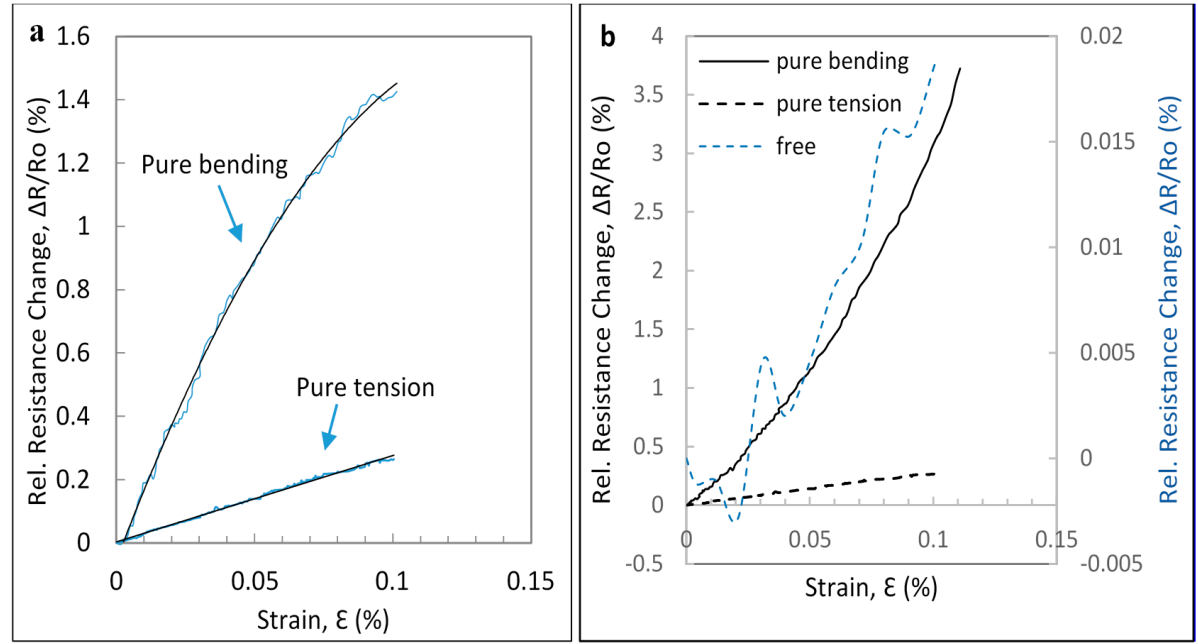

Figure 8. Effect of lateral constraint on the piezoresistive response of free and constrained CNT yarns. Relative resistance change-strain curves: (a) $0.1 \%$-maximum strain $\left(0.003 \mathrm{~min}^{-1}\right)$; (b) $0.1 \%$-maximum strain $\left(0.006 \mathrm{~min}^{-1}\right)$.

Table 2. Average sensitivity (strain gauge factor) of three constrained and free CNT yarns in terms of strain rate $\left(\mathrm{min}^{-1}\right)$.

\begin{tabular}{|c|c|c|c|c|}
\hline Strain Rate $\left(\min ^{-1}\right)$ & Strain $(\%)$ & $\begin{array}{l}\text { Gauge Factor (Constrained } \\
\text { CNT Yarn-Pure Bending) }\end{array}$ & $\begin{array}{l}\text { Gauge Factor (Constrained } \\
\text { CNT Yarn-Pure Tension) }\end{array}$ & Gauge Factor (Free CNT Yarn) \\
\hline 0.006 & 0.05 & 22.02 & 3.11 & 0.12 \\
\hline 0.003 & 0.05 & 13.48 & 2.56 & 0.11 \\
\hline 0.006 & 0.1 & 26.74 & 2.83 & 0.20 \\
\hline 0.003 & 0.1 & 15.12 & 2.72 & 0.16 \\
\hline
\end{tabular}

From previous research, the strain rate of $0.006 \mathrm{~min}^{-1}$ is considered a high quasi-static rate and it was selected in this study [12]. It is assumed that the distance between the carbon atoms are longer under tension. This leads to an increase in the resistance. At high strain rates, this phenomenon is dominant in the free CNT yarns and consequently an increase in resistance is observed. At low strain rates, fiber slippage is high leading to a decrease in the resistance. Similarly, an increase in the resistance is also observed in the case of constrained CNT yarns under tension and this increase is even higher than that of unconstrained CNT yarns. Therefore, based on all previous results and those of this study, the following hypotheses are proposed. Because of the lateral effect exerted by the polymer host medium, the cross section of the CNT yarns cannot shrink but their length can increase along with the length of polymeric beam. Hence, fiber slippage is restricted when the CNT yarn is embedded in polymeric beam. This leads to a significant increase in the resistance of the constrained CNT yarns.

\section{Conclusions}

The piezoresistive response of laterally-constrained CNT yarns was determined for the first time. The rationale of this study was to mimic the piezoresistive response of CNT yarn sensors that are integrated in polymers or composites. The response of unconstrained CNT yarns under tension had been determined 
previously and the results could now be compared with those in this study. All these results were obtained using quasi-static strain rates. It is very important to note that the previous results had shown that the strain rate plays a very significant role not only on the amount of piezoresistivity in the free CNT yarn but in the sign of the piezoresistivity and its response varying from quasi-parabolic to linear. By subjecting a CNT yarn embedded into a polymer medium to four-point bending and uniaxial tension, the piezoresistive response of the constrained CNT yarns was determined under tension and compression. The relative resistance change of both the constrained and unconstrained CNT yarn increases monotonically with the strain. However, the piezoresistive response of the constrained CNT yarns is much more sensitive than that of the unconstrained CNT yarns. This difference between them may be explained by the lack of the effective slippage, fiber unraveling and subsequently, Poisson's effect, of the CNT yarn when integrated in the polymer. The composite samples tested under bending showed a higher gauge factor than under uniaxial tension. Higher sensitivity was observed for the samples tested at higher strain rates with gauge factors increasing with increasing strain levels. The negative piezoresistivity experienced by a free CNT yarn under low strain rate conditions was not encountered at similar strain rates for the constrained CNT yarn. Therefore, a foregone conclusion is that slippage plays a deeper role in the resistance decrease of CNT yarns upon loading at low strain rates.

The piezoresistive response of the constrained CNT yarns under compression was observed to exhibit a quasi-parabolic response. Like in the tensile tests, the relative change in resistance decreases slightly at first reaching a local minimum and tends to increase later. The hypotheses that were used to explain the phenomena of the CNT yarn under tension could also be applied in the case of the constrained CNT yarns under compression. Increased fiber density means charge carriers becomes closer and the resistance decreases. The relative change in resistance also decreases. Therefore, a higher gauge factor is obtained under tension than under compression.

Acknowledgments: The authors thank all the students in the Intelligent Materials laboratory at The Catholic University of America for their logistical support to this study. Jandro L. Abot acknowledges the logistical support of Kalayu Belay at the Florida Agricultural and Mechanical University and the financial support from the Air Force Office of Scientific Research (AFOSR) through Grants FA9550-10-1-0040 and FA9550-15-1-0177, both with program officer David Stargel and more recently, James Fillerup and Lt. Col. Jamie Morrison, and from the National Aeronautics Space Administration (NASA) District of Columbia Space Grant Consortium (DCSGC) through Grant 31154.

Author Contributions: Jude C. Anike, Huy H. Le, Grace E. Brodeur and Mathew M. Kadavan conducted the experimental sections of the study. Jude C. Anike and Jandro L. Abot co-wrote the paper.

Conflicts of Interest: The authors declare no conflict of interest.

\section{References}

1. Abot, J.L.; Rajan, C.P. Carbon nanotube fibers. In Carbon Nanomaterials Sourcebook: Graphene, Fullerenes, Nanotubes, and Nanodiamonds; Sattler, K.D., Ed.; Taylor and Francis: London, UK, 2016; Volume I, pp. $357-383$.

2. Zhao, J.; Zhang, X.; Di, J.; Xu, G.; Yang, X.; Liu, X.; Yong, Z.; Chen, M.; Li, Q. Double-peak mechanical properties of carbon-nanotube fibers. Small 2010, 6, 2612-2617. [CrossRef] [PubMed]

3. Zhao, Y.; Wei, J.; Vajtai, R.; Ajayan, P.M.; Barrera, E.V. Iodine doped carbon nanotube cables exceeding specific electrical conductivity of metals. Sci. Rep. 2011, 1, 83. [CrossRef] [PubMed]

4. Zhang, M.; Atkinson, K.R.; Baughman, R.H. Multifunctional carbon nanotube yarns by downsizing an ancient technology. Science 2004, 306, 1358-1361. [CrossRef] [PubMed]

5. Liu, L.; Ma, W.; Zhang, Z. Macroscopic carbon nanotube assemblies: Preparation, properties, and potential applications. Small 2011, 7, 1504-1520. [CrossRef] [PubMed]

6. Zhang, S.; Koziol, K.K.K.; Kinloch, I.A.; Windle, A.H. Macroscopic fibers of well-aligned carbon nanotubes by wet spinning. Small 2008, 4, 1217-1222. [CrossRef] [PubMed]

7. Ma, W.; Song, L.; Yang, R.; Zhang, T.; Zhao, Y.; Sun, L.; Ren, Y.; Liu, D.; Liu, L.; Shen, J.; et al. Directly synthesized strong, highly conducting, transparent single-walled carbon nanotube films. Nano Lett. 2007, 7, 2307-2311. [CrossRef] [PubMed] 
8. Ma, W.; Liu, L.; Yang, R.; Zhang, T.; Zhang, Z.; Song, L.; Ren, Y.; Shen, J.; Niu, Z.; Zhou, W.; et al. Monitoring a micromechanical process in macroscale carbon nanotube films and fibers. Adv. Mater. 2009, 21, 603-608. [CrossRef] [PubMed]

9. Ma, W.; Liu, L.; Zhang, Z.; Yang, R.; Liu, G.; Zhang, T.; An, X.; Yi, X.; Ren, Y.; Niu, Z.; et al. High-strength composite fibers: Realizing true potential of carbon nanotubes in polymer matrix through continuous reticulate architecture and molecular level couplings. Nano Lett. 2009, 9, 2855-2861. [CrossRef] [PubMed]

10. Liu, K.; Sun, Y.; Zhou, R.; Zhu, H.; Wang, J.; Liu, L.; Fan, S.; Jiang, K. Carbon nanotube yarns with high tensile strength made by a twisting and shrinking method. Nanotechnology 2010, 2, 045708. [CrossRef] [PubMed]

11. Abot, J.L.; Alosh, T.; Belay, K. Strain dependence of electrical resistance in carbon nanotube yarns. Carbon 2014, 70, 95-102. [CrossRef]

12. Anike, J.C.; Bajar, A.; Abot, J.L. Time-dependent effects on the coupled mechanical-electrical response of carbon nanotube yarns under tensile loading. Carbon 2016. [CrossRef]

13. Cullinan, M.; Culpepper, M. Carbon nanotubes as piezoresistive microelectromechanical sensors: Theory and experiment. Phys. Rev. B 2010, 82, 115428. [CrossRef]

14. Lekawa-Raus, A.; Koziol, K.K.K.; Windle, A.H. Piezoresistive effect in carbon nanotube fibers. ACS Nano 2014, 8, 1114-1124. [CrossRef] [PubMed]

15. Zhao, H.; Zhang, Y.; Bradford, D.; Zhou, Q.; Jia, Q.; Yuan, G.; Zhu, Y. Carbon nanotube yarn strain sensors. Nanotechnology 2010, 21, 305502. [CrossRef] [PubMed]

16. Anike, J.C.; Belay, K.; Williams, M.E.; Abot, J.L. Piezoresistive response of carbon nanotube yarns under tension: Rate effects and phenomenology. New Carbon Mater. 2017, under review.

17. Alvarez, N.T.; Miller, P.; Haase, M.; Kienzle, N.; Zhang, L.; Schulz, M.J.; Shanov, V. Carbon nanotube assembly at near-industrial natural-fiber spinning rates. Carbon 2015, 86, 350-357. [CrossRef]

18. ASTM International. Standard Test Method for Tensile Properties of Plastics; ASTM D638; ASTM International: West Conshohocken, PA, USA, 2014.

19. Miao, M. Yarn spun from carbon nanotube forests: Production, structure, properties and applications. Particuology 2013, 11, 378-393. [CrossRef]

20. Thiagarajan, V.; Wang, X.; Bradford, P.D.; Zhu, Y.T.; Yuan, F.G. Stabilizing carbon nanotube yarns using chemical vapor infiltration. Compos. Sci. Technol. 2014, 90, 82-87. [CrossRef]

21. Schulz, M.; Shanov, V.; Yin, Z. Nanotube Superfiber Materials: Changing Engineering Design; Elsevier: Oxford, UK, 2014.

22. Lu, W.; Zu, M.; Byun, J.-H.; Kim, B.-S.; Chou, T.-W. State of the art of carbon nanotube fibers: Opportunities and challenges. Adv. Mater. 2012, 24, 1805-1833. [CrossRef] [PubMed]

23. Jayasinghe, C.; Li, W.; Song, Y.; Abot, J.L.; Shanov, V.N.; Fialkova, S. Nanotube responsive materials. MRS Bull. 2010, 35, 682-692. [CrossRef]

24. Alosh, T. Electrical Resistivity Dependence on Mechanical Stresses in Carbon Nanotube Yarns. Master's Thesis, The Catholic University of America, Washington, WA, USA, 2013.

25. Alvarez, N.T.; Ochmann, T.; Kienzle, N.; Ruff, B.; Haase, M.R.; Hopkins, T.; Pixley, S.; Mast, D.; Schulz, M.J.; Shanov, V. Polymer coating of carbon nanotube fibers for electric microcables. Nanomaterials 2014, 4, 879-893. [CrossRef] [PubMed]

26. Vilatela, J.J.; Khare, R.; Windle, A.H. The hierarchical structure and properties of multifunctional carbon nanotube fibre composites. Carbon 2012, 50, 1227-1234. [CrossRef]

27. Rai, A.K.; Porwal, A.; Mishra, S.B. Investigation of mechanical properties of epoxy EPON 862 cured with TETA by molecular dynamics. S-JPSET 2013, 4, 2. [CrossRef]

28. Wang, X.; Chung, D.D.L. Short-carbon-fiber-reinforced epoxy as a piezoresistive strain sensor. Smart Mater. Struct. 1995, 4, 363-367. [CrossRef]

29. Abot, J.L.; Schulz, M.J.; Song, Y.; Medikonda, S.; Rooy, N. Novel distributed strain sensing in polymeric materials. Smart Mater. Struct. 2010, 19, 085007. [CrossRef]

(c) 2017 by the authors. Licensee MDPI, Basel, Switzerland. This article is an open access article distributed under the terms and conditions of the Creative Commons Attribution (CC BY) license (http:/ / creativecommons.org/licenses/by/4.0/). 